\title{
LEARNING IN LARGE OPTICAL NETWORKS
}

\author{
Yong Qiao and Demetri Psaltis \\ California Institute of Technology \\ Department of Electrical Engineering \\ Pasadena, CA 91125
}

\begin{abstract}
In a holographic optical learning network, the decay of multiply exposed holographic interconnections can adversely affect the training of the network. A new dynamic photorefractive holographic memory is described that allows an arbitrarily long sequence of adaptations by rejuvenating decayed holograms with a simple all-optical feedback loop.
\end{abstract}

\section{INTRODUCTION}

The two key components in an optical neural network are the neuron arrays and the interconnections between neurons. While neuron arrays can be implemented by spatial light modulators and more recently by optoelectronic smart pixel arrays, volume holograms provide an effective way of realizing massive, parallel interconnections by storing information in 3-D [1]. One of the most interesting features of a neural network is its ability to learn. Learning is achieved by modifying the weights of interconnections in a supervised or unsupervised fashion. In optical learning networks, this requires that the holographic interconnections can be dynamically changed in real time. Photorefractive crystals are one of the very promising materials for realizing modifiable holographic interconnections [2-4]. During each learning iteration, the required weight change is calculated according to some algorithm, and then the change is made to the photorefractive hologram by exposing it to light.

For large optical networks, many iterations would be expected for them to learn a desired mapping. This means that many exposures would have to be made to a photorefractive hologram. When a new exposure is made to a photorefractive crystal, the previously recorded hologram will decay. If we want all the exposures to have equal contribution to the final hologram, an exposure schedule [2] has to be followed in which the time for more recent exposure has to be shorter than that for the previous ones. The consequence is that the diffraction efficiency of the hologram becomes inversely proportional to the number of exposures used to form the hologram, if these exposures are statistically independent [2]. In other words, the diffraction efficiency will rapidly decrease with the increase in the number of learning iterations. Therefore the number of learning iterations that a photorefractive hologram can provide is eventually limited by the background noise in the system. On the other hand, if we use the same time for all the exposures rather than following the exposure schedule, then previous exposures will contribute less than more recent ones and this may slow down the learning convergence or even cause the system not to converge.

\section{DYNAMIC COPYING}

One method to overcome the hologram decay problem is dynamic copying [5-8]. The basic idea is to transfer the multiply exposed hologram to a second medium, and then copy it back with a single exposure to rejuvenate the primary hologram. As a result, the overall diffraction efficiency after copying becomes independent of the number of holographic exposures used to form the original hologram. Here a new dynamic holographic memory system is described. The system, shown in Fig. 1, consists of a single crystal for storing adaptive holograms and an optical feedback loop with a liquid crystal light valve (LCLV). The 
input patterns are displayed on a spatial light modulator, imaged on to the writing side of the LCLV, and read out from the other side. In addition, the LCLV performs optical amplification and thresholding in the loop. When a hologram becomes weak after multiple exposures, the feedback loop is closed and the reference beam reconstructs the hologram. The reconstructed signal (which is a 2-D pattern) is fed back to the writing side of the LCLV, and quickly amplified by the LCLV and the feedback loop. The original hologram is then refreshed by rewriting it with the amplified reconstructed signal.

The optical feedback loop shown in Fig. 1 is inherently unstable. Any perturbation in the feedback signal due to misalignment in the loop will be amplified, resulting in a smeared feedback signal and a smeared refreshed hologram. A novel feature of this dynamic memory system is the use of spatial sampling for stabilizing the system. The idea is to spatially sample the inputs and the feedback patterns by illuminating the reading side of the LCLV with a 2-D light spot array. When the reconstructed signal is fed back to the writing side of the LCLV, each of the pixels is magnified by placing the LCLV slightly before the image plane of the feedback signal. Therefore any misalignment between the input and feedback patterns will be corrected as long as the reading-side sampling spot does not miss the magnified pixel of the feedback signal. In the experiment, an $128 \times 128$ microlenslet array and a 4-f system was used to generate the sampling spot array. Fig. 2(a) shows the dynamically stored hologram when the initial one was recorded with a single exposure of the letter $A$ and the sampling was used. The quality of the stored image remained virtually unchanged during the dynamic copying. Fig. 2(b) shows the same thing except that the sampling was removed. For the unsampled case, careful alignment simply slowed the transition from a recognizable image to the smeared final state.

An important property of this dynamic memory is that the relative phases of the stored holograms are maintained during copying. This is true because any of the holograms is recorded and copied with the same reference and signal beams that have a fixed phase relationship. In the case of hologram formation by diffusion only (such for the $\mathrm{BaTiO}_{3}$ crystal used in our experiment), the consequence is that the hologram will always remain phase locked. This property is crucial for optical neural networks, where the phases of the holograms must be accurately controlled so that each holographic interconnection can be selectively enhanced or reduced.

To realize parallel, independent interconections from one neuron array to another, multiple holograms associated with different reference beams will have to be recorded. This is done in the system of Fig. 1 by changing the angle of the reference beam with a mirror mounted on a motorized rotary stage. These holograms are dynamically enhanced by scanning the angle of the reference beam, sequentially reading out and rejuvenating each stored hologram, and repeating this cycle many times.

An arbitrary hologram can be selectively enhanced or reduced by changing the phase of the corresponding reference beam with an electrically controlled liquid crystal phase retarder. Using dynamic copying after each or several holographic exposures, multiple holograms can be continuously updated without losing their strength.

In summary, an experimental dynamic holographic memory is presented here. This approach allows us to construct optical learning networks capable of an arbitrarily long sequence of adaptations.

\section{ACKNOWLEDGEMENTS}

This work was supported by DARPA and the Air Force Office of Scientific Research.

\section{REFERENCES}

1. D. Psaltis, D. Brady, X. -G. Gu, and S. Lin, "Holography in artificial neural networks," Nature 343, 325 (1990).

2. D. Psaltis, D. J. Brady, and K. Wagner, "Adaptive optical networks using photorefractive crystals," 
Appl. Opt. 27, 1752 (1988).

3. D. Z. Anderson and D. M. Lininger, "Dynamic optical interconnects: volume holograms as optical two-port operators," Appl. Opt. 26, 5031 (1987).

4. E. G. Paek, J. R. Wullert, and J. S. Patel, "Holographic implementation of a learning-machine based on a multicategory perceptron algorithm," Opt. Lett. 14, 1303 (1989).

5. D. Brady, K. Hsu and D. Psaltis, "Periodically refreshed multiply exposed photorefractive holograms," Opt. Lett. 15, 817 (1990).

6. Y. Qiao, D. Psaltis, C. Gu, J. Hong, P. Yeh, and R. R. Neurgaonkar, "Phase-locked sustainment of photorefractive holograms using phase conjugation," J. Appl. Phys. 70, 4646 (1991).

7. H. Sasaki, Y, Fainman, J. E. Ford, Y. Taketomi, and S. H. Lee, "Dynamic photorefractive optical memory," Opt. Lett. 16, 1874 (1991).

8. S. Boj, G. Pauliat, and G. Roosen, "Dynamic holographic memory showing readout, refreshing, and updating capabilities," Opt. Lett. 17, 438 (1992).

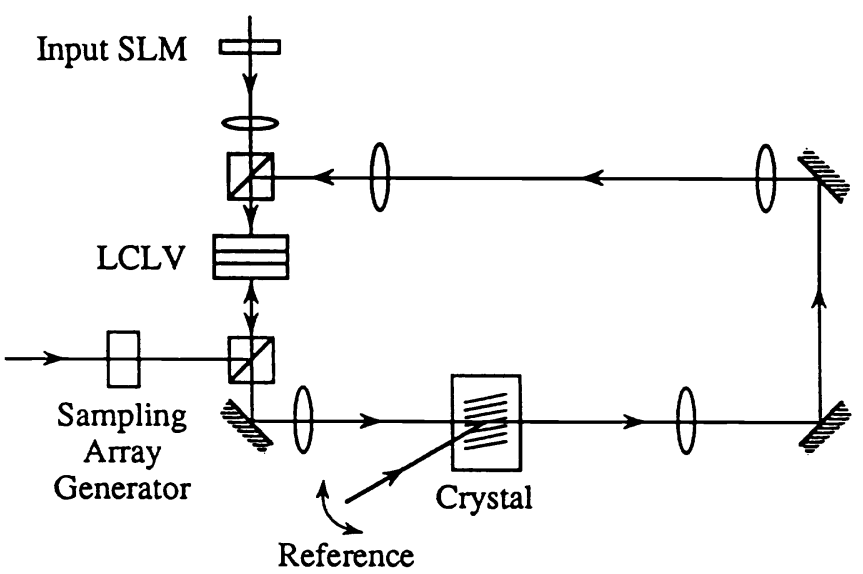

Figure 1. Schematic diagram of the sampled dynamic holographic memory.

(a)

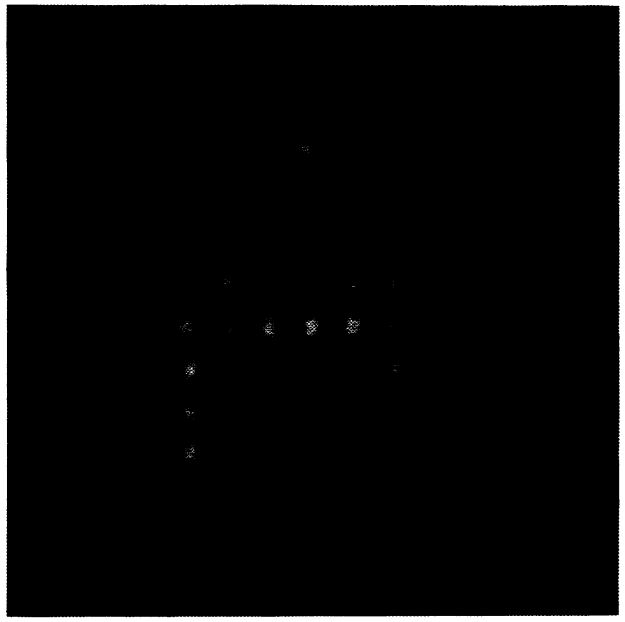

(b)

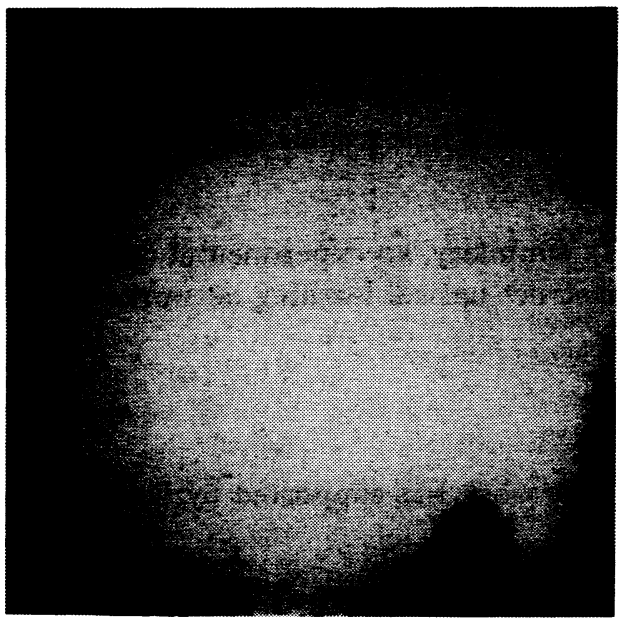

Figure 2. Examples of images dynamically stored in the crystal: (a) with sampling; (b) without sampling. 